\title{
Orthosis Design System for Malformed Ears Based on Spline Approximation
}

\author{
Akihiko Hanafusa $^{1}$, Tsuneshi Isomura ${ }^{1}$, Yukio Sekiguchi ${ }^{1}$, \\ Hajime Takahashi ${ }^{2}$, and Takeyoshi Dohi ${ }^{3}$ \\ ${ }^{1}$ Department of Rehabilitation Engineering, Polytechnic University \\ 4-1-1 Hashimotodai, Sagamihara, Kanagawa 229-1196, Japan \\ hanafusa@uitec.ac.jp \\ ${ }^{2}$ Department of Plastic Surgery, Tokyo Metropolitan Toshima Hospital \\ 33-1 Sakaemachi, Itabashi-ku, Tokyo 173-0015, Japan \\ hajime-t@toshima-hp.metro.tokyo.jp \\ ${ }^{3}$ Graduate School of Information Science and Technology, The University of Tokyo \\ 7-3-1 Hongou, Bunkyou-ku, Tokyo 113-8654, Japan \\ dohi@miki.pe.u-tokyo.ac.jp
}

\begin{abstract}
Malformed ears of neonates can be effectively treated by employing an orthosis of suitable shape. Currently, we use orthoses made of nitinol shape memory alloy wire and have developed a computer-assisted design system to manufacture the orthosis. Using this method, extracted contours of the helix and auriculotemporal sulcus are approximated to spline, and orthosis shape can be designed by moving the control points of spline with reference to control points of the target auricular shape. The system also functions to evaluate the contact force between the orthosis and auricle. Using this system, orthoses were designed and manufactured for 16 patients with malformed ears. Treatment was more effective in cases where it was necessary to extend the helix.
\end{abstract}

\section{Introduction}

In Japan, approximately $20 \%$ of neonates are born with an auricular deformity that will not heal spontaneously. Most cases can be treated by mounting an appropriately shaped orthosis in the auricle [1]. Currently, the authors use orthoses made of nitinol shape memory alloy wire covered with an expanded polytetrafluoroethylene tube. An example of treatment of a folded helix using an orthosis is illustrated in Fig.1. For manufacture of the orthosis, the wire is fixed in the appropriate shape and the shape is memorized by heating to $500^{\circ} \mathrm{C}$ for 30 minutes. An iron plate is grooved by the shape of the orthosis and the wire is inserted to fix the shape. We have recently developed a computer-assisted design system [2] for use in constructing the orthosis. Previously, there have been only limited attempts to employ such a system for this purpose. One example is a system that constructs a wax auricular model using three-dimensional shape measuring system and numerically controlled machine tool. This has been used for planning of a microtia operation [3].

Here, we describe a newly developed orthosis design method that focuses on post therapeutic auricular shape, and orthosis shape is generated based on a splineapproximated curve. The system also permits an estimation of the contact force between the auricle and orthosis by finite element analysis. This system was developed using MATLAB (The Math Works Inc.), and we have introduced several clinical 
applications of the system. In each case, treatment was performed only after thorough explanation of the procedure by the physician, and with the agreement of the patients' parents.

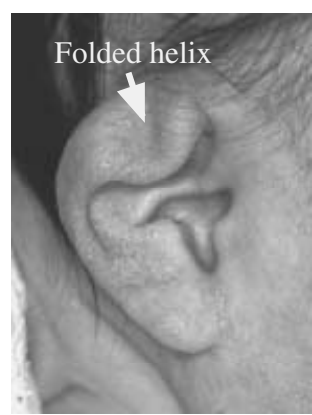

(a) Before treatment

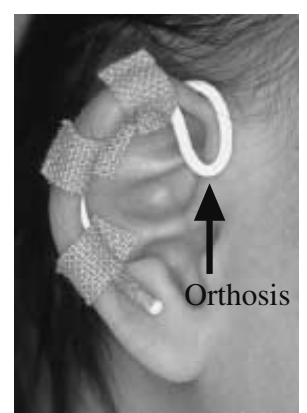

(b) Orthosis mounted in the auricle during treatment

Fig. 1. A case of folded helix, and treatment using an orthosis made of nitinol shape memory wire

\section{Spline Approximation of Auricular Shape [4]}

Spline curves are widely used in the field of CAD/CAM and computer graphics, with B-splines being the most popular in these applications. To approximate auricular shape in the current investigation, B-spline function of order four with four internal knots is used. An initial step is to convert the co-ordinates of auricular contour points. This conversion is based on the ear base line, that is a line connecting Otobasion superius $(o b s)$ and Otobasion inferius (obi), as illustrated in Fig.2(a). In addition, the distance is normalized based on the length of the line between $O$ and $o b s$, thus permitting the comparison of various auricle shapes. $X$ and $Y$ co-ordinates of contour points are defined by the medium of $\theta$, and approximated co-ordinates are defined by the B-spline function as shown in the equation (1).

$$
x(\theta)=!_{i=1}^{8} \alpha_{i} B_{i, 4}(\theta) \quad y(\theta)=!_{i=1}^{8} \beta_{i} B_{i, 4}(\theta)
$$

Coefficients $\left(\alpha_{i}, \beta_{i}\right)$ are calculated using the least square method and plotted on the $X Y$ plane as control points $(C P i)$. The position of the control points can then be used as an indicator of auricular shape.

Using photographs, we examined the auricular shape of 550 ears of Japanese neonates under the age of 7 days, and classified them as normal or abnormal (five categories). Fig.2(b) illustrates the distribution of control points for 130 normal ears.

The large X's are the average normal control points, that is the average position of control points for normal ears, and the thick curve represents the average shape of a normal ear as determined by the position of the average normal control points. When an individual control point is specified, Mahalanobis generalized distance and the probability of belonging to the group can be calculated by the distribution of control points. We derive the normal rate by the ratio of probability of normal group and that of abnormal groups. 


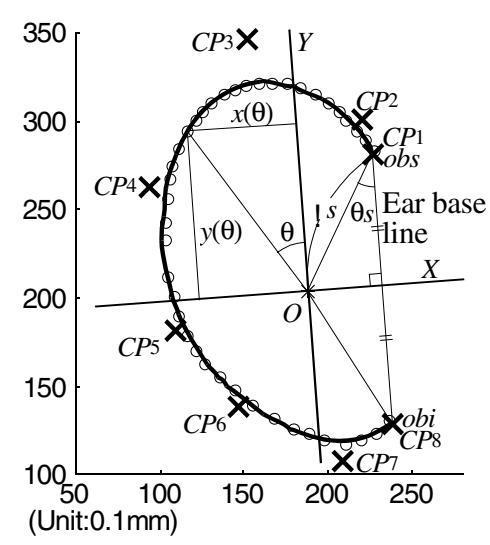

(a) Axes of co-ordinates.

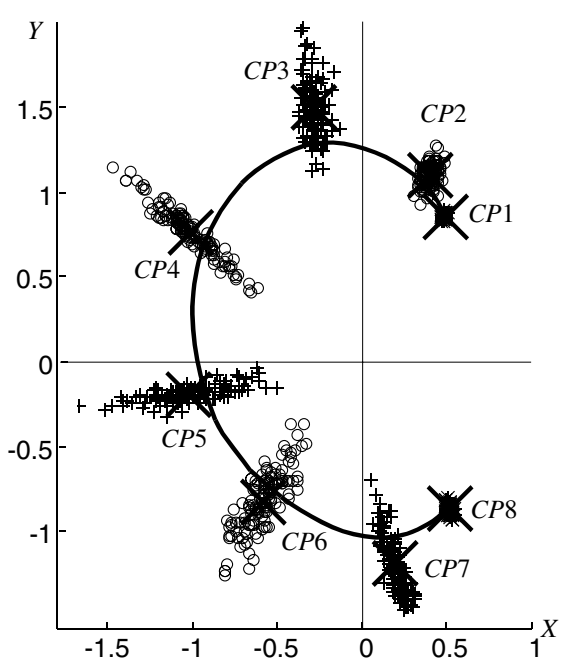

(b) Distribution of control points and average shape of normal ear.

Fig. 2. Approximated spline of an auricle

\section{Generation of Orthosis Shape}

An Orthosis shape is generated as follows:

1) An auricular three-dimensional model is composed;

2) Contours of the helix and auriculotemporal sulcus are obtained;

3) Contours are approximated by spline and the position of control points are compared with those of target post therapeutic auricular shapes. The control points are moved toward the target positions; and

4) The orthosis shape and output manufacturing data are generated.

\subsection{Composure of the Auricular Three-Dimensional Model}

The three dimensional auricular shape is measured using the non-contact laser measurement system, which can acquire not only three dimensional position data, but also RGB color data. Since it is not possible to acquire data from both the frontal side of the auricle and the rear side (including the auriculotemporal sulcus) at the same time, it is necessary to compose a three-dimensional image using data obtained from measurements made from various directions.

In order to improve the accuracy of auricular matching, we use a composite method that can match not only the surface contour but also the color. This method is based on the Iterative Closest Point (ICP) algorithm [5], with improvements to permit handling of both three-dimensional and RGB color co-ordinate distance. Equation (2) shows the defined united distance $(d)$ of three dimensional distance and color distance. Here, $P_{a}, P_{b}, C_{a}, C_{b}$ are three-dimensional co-ordinates and color coordinates of points $a$ and $b$ respectively, and $k p$ and $k c$ are weighted coefficients. 
Usually, a plaster cast of the auricle, colored in a striped pattern, is used to compose the three dimensional model and color distance is used auxiliarily be setting coefficients to $k p=0.9$ and $k c=0.1$.

$$
d^{2}=k p^{2}\left|P_{a}-P_{b}\right|^{2}+k c^{2}\left|C_{a}-C_{b}\right|^{2}
$$

\subsection{Extraction of Contours of the Helix and Auriculotemporal Sulcus}

Fig. 3 shows the triangular element modification and line segment extraction system. The system includes a function to trace to the next point, situated where the difference of curvature and direction of normal vector from the current point is smallest. Contours can be obtained by selecting the Otobasion superius $(o b s)$, and tracing either helix or auriculotemporal sulcus points, automatically or manually, to the Otobasion inferius $(o b i)$.

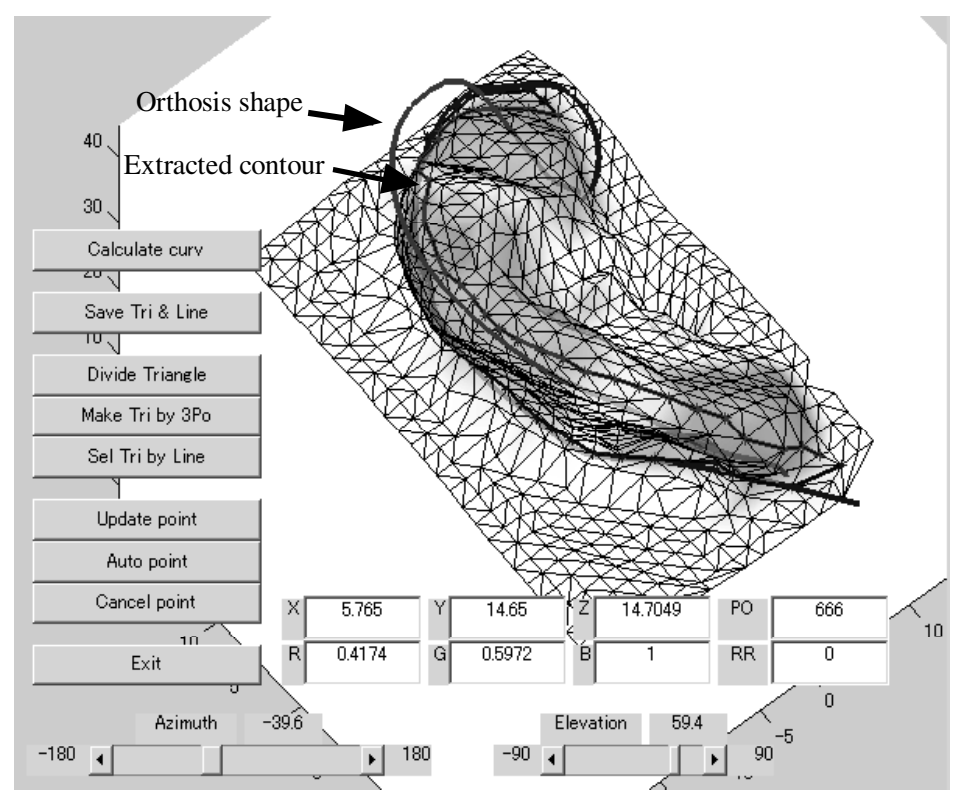

Fig. 3. Overlaid display of generated orthosis and auricular model

\subsection{Approximation of Contours by Spline and Comparison of Control Points}

To fix and memorize the orthosis shape to the shape memory alloy wire, the helix and the auriculotemporal sulcus side plates are grooved separately. The approximated plane is first calculated and co-ordinates of points on the contour are projected onto the plane. Approximated spline is then calculated using the method described in the previous section. Subsequently, the current position of control points and the position of control points for the post therapeutic target shape are compared. As a post therapeutic target shape, spline approximation results of normal ear shape, obtained from a 
normal ear on the opposite side or from a sibling or parent, can be used. Also, the average position of normal ear control points can be employed. By moving the position of control points, shape of contours can be modified.

\subsection{Generation of the Orthosis Shape}

In accordance with the modified shape of the contours, the orthosis shape is generated. Fig.3 illustrates an overlaid display of a generated orthosis and auricular model. Finally, the shape is converted to Numerical Control (NC) data for groove process using a machine tool.

\section{Finite Element Analysis of Contact Force [6]}

It is important to evaluate the contact force between the orthosis and auricle, to ensure that it is sufficient to correct the auricle shape, while not excessive to the degree that it may cause a decubitus-like inflammation on the auricle. To evaluate the contact force, finite element analysis, that can handle the material non-linearity of an auricle and the contact deformation of auricle and orthosis, is developed.

To contend with the material non-linearity, an incremental method is employed and displacement is increased by inserting the orthosis gradually. Moreover, we have also performed a tensile test using pig's auricular cartilage, and applied the resultant strain-stress diagram to the material property. Multiple point constraints are also used to represent the contact deformation of auricle and orthosis. The constraint condition should be updated in every insertion step of the incremental method.

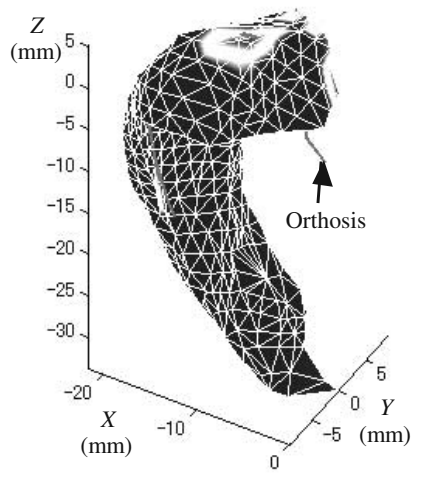

(a) Control points lie halfway between their original position and that of the average control points in a normal situation

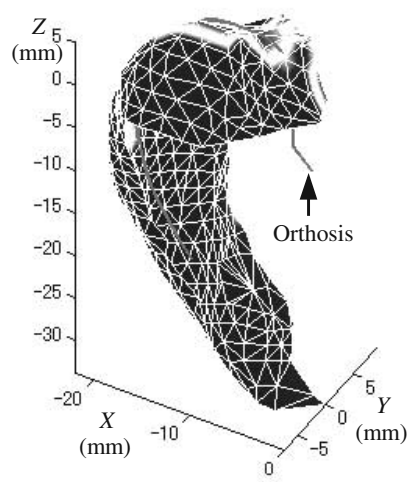

(b) Control points are moved to the position of average control points in a normal situation

Fig. 4. Simulation results to demonstrate the effect of moving control points on the contact force distribution on the auricle

Where an orthosis was used in the case of an upper folded helix, as illustrated in Fig.1, contact force was compared for different positions of control points. Figs.4(a) and 5(a) illustrate the outcome of moving control points $C P 2$ to $C P 4$ (see Fig. 2(b)) halfway towards the position of average normal control points as illustrated in 
Fig.6(a) (middle line). Figs.4(b) and 5(b) illustrate the effect of moving $C P 2$ to $C P 4$ to the position of average normal control points. Orthoses of helix side are inserted in 8 steps, increasing by $0.5 \mathrm{~mm}$ each, and the contact force is evaluated. The start edge of the orthosis and auriculotemporal sulcus of the auricle are clipped. The number of elements of the auricle is 630, and that of orthosis is 28. Fig.4 shows the distribution of the force on the auricle. Brighter area where more force is implied is increased when the average of normal position is used. Fig.5 illustrates orthosis deformation and the force produced by the contact. When the control points are in the middle of current and average normal position, the force at the clip point is 0.63 times smaller and the maximum contact force is 0.67 times smaller.

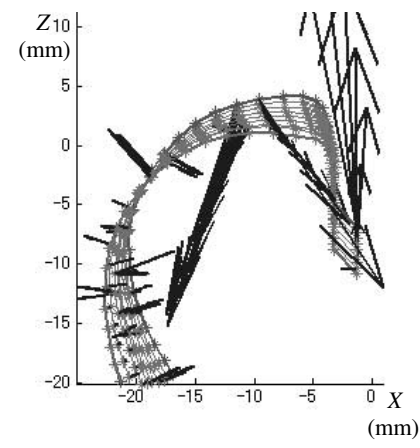

(a) Control points lie halfway between their original position and that of the average control points in a normal situation

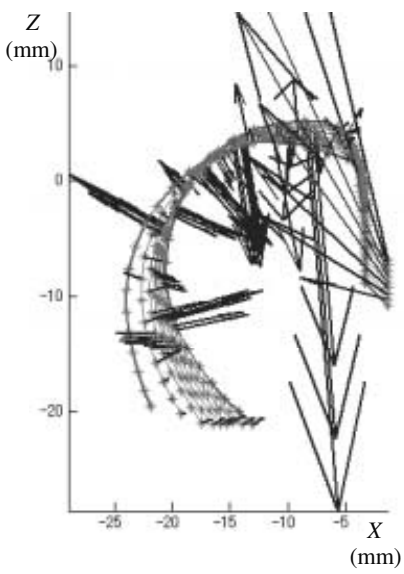

(b) Control points are moved to the position of average control points in a normal situation

Fig. 5. Simulation results to demonstrate the effect of moving control points on orthosis deformation and contact force on the orthosis

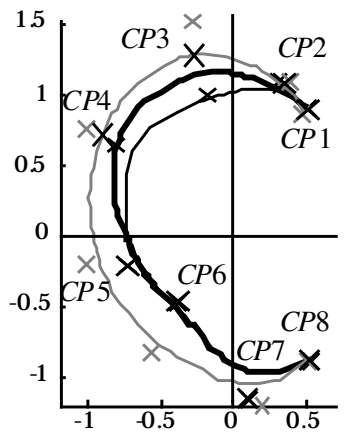

(a) Control points used to design the orthosis

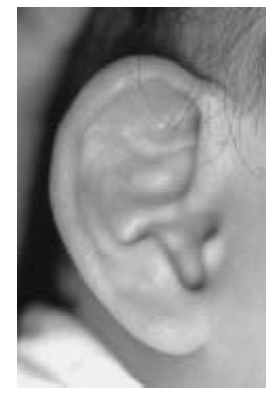

(b) Clinical result after four months of treatment

Fig. 6. Treatment of the folded helix case shown in Fig.1 


\section{Clinical Applications}

The clinical application of nitinol orthoses, designed and manufactured using the recently-developed system, was evaluated in 16 cases. More specifically, this study set included 6 cases of cryptotia, 5 cases of folded helix, 3 cases of folded lobulus auriculae, and one case of Stahl's ear and one case of a protruded auricula. Currently 11 of these cases are under treatment and effectiveness of treatment has been confirmed in 9 cases. Where treatment required extension of the helix, employing this method generally resulted in improvement. However, where it was necessary to form anthelicis as part of treatment, it was impossible to effectively treat the deformity using only the developed orthosis.

\subsection{Treatment of a Folded Helix}

Fig.1(a) show a folded helix in a one-month-old baby. Fig.6(a) shows the spline data used to generate the orthosis for this case. From the center of the figure outwards, the three lines represent the approximated spline of auricle pre-treatment, orthosis shape and the average shape of a normal ear respectively. Control points $C P 2, C P 3$ and $C P 4$ are moved halfway between the current position and position of average normal points. Fig.1(b) shows the orthosis mounted in the auricle and, as illustrated in Fig.6(b), after 4-months of treatment the degree of folding was improved.

\subsection{Treatment of a Folded Lobulus-Auriculae}

Figs.7 and 8 illustrate the use of the system to correct a folded lobulus-auriculae in a three-month-old baby (Fig.7(a)). The orthosis shape shown in Fig.7(b) is generated by moving control points $C P 4, C P 7$ and $C P 8$ toward the normal average position, as demonstrated in Fig.8(a). After one month, the degree of folding was improved (Fig.8(b)).

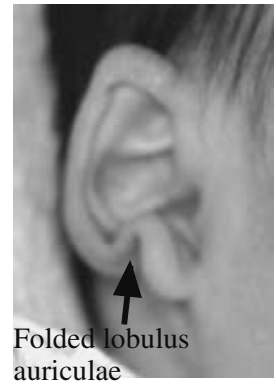

(a) Before treatment

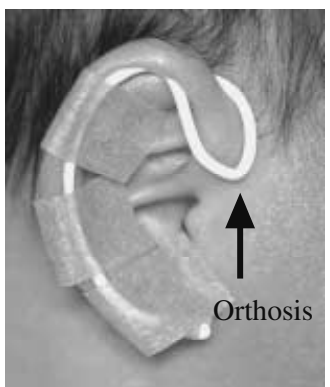

(b) Orthosis mounted in the auricle during treatment

Fig. 7. A case of folded lobulus-auriculae, and treatment using an orthosis 


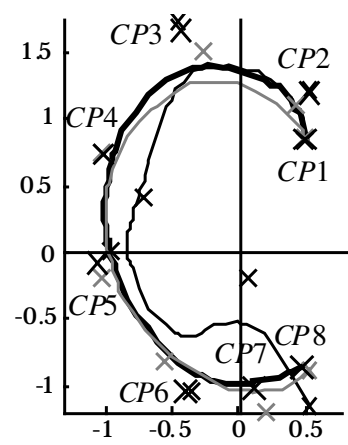

(a) Control points used to design the orthosis

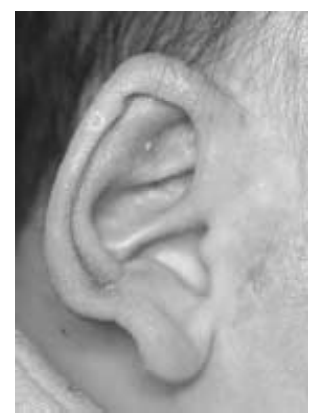

(b) Clinical result after one month of treatment

Fig. 8. Treatment of the folded lobulus-auriculae case shown in Fig.7

\section{Conclusion}

Using the orthosis design system, a three dimensional auricular model can be produced that considers both surface contour and color. Extracted contours of the helix and auriculotemporal sulcus are approximated to spline, and orthosis shape can be designed by moving the control points of spline, referring to control points of the target auricular shape.

The system also permits evaluation of the contact force between the orthosis and auricle. By moving control points halfway towards the position of average normal control points, the contact force is less than that associated with moving control points to the average normal positions.

Orthoses for 16 cases of malformed ears were designed and manufactured using the system, and treatment was effective in 9 cases when the helix had to be extended.

\section{References}

1. Matsuo K, Hirose T, Tomono T, Iwasawa M, Katohda S, Takahashi N, Koh B : Nonsurgical Correction of Congenital Auricular Deformities in the Early Neonate, Plast. Reconstr. Surg. 73 (1984) 38-50.

2. Hanafusa A, Takahashi H, Akagi K, Isomura T : Development of Computer Assisted Orthosis Design and Manufacturing System for Malformed Ears, Computer Aided Surgery 2 (1997) 276-285.

3. Kaneko T.: A System for Three-Dimensional Shape Measurement and its Application in Microtia Ear Reconstruction, Keio J. Med. 42(1) (1993) 22-40.

4. Hanafusa A, Takahashi H, Isomura T, Dohi T: Analyses of Japanese Neonates' Auricular Shape Using Spline Approximation, Proc. of the CAR'98 (1998) 951.

5. Besl PJ, McKay ND : A Method for Registration of 3-D Shapes, IEEE Trans. Pattern Analysis and Machine Intelligence, 14, 2 (1992) 239-256.

6. Hanafusa A, Isomura T, Sekuguchi Y, Takahashi H, Dohi T: Computer Assisted Orthosis Design System for Malformed Ears -Automatic Shape Modification Method for Preventing Excessive Corrective Force-, Proc. of the World Congress on Medical Physics and Biomedical Engineering Chicago 2000 (2000) 1-3. 